\title{
A Composite Control Strategy for an Air-Breathing Hypersonic Vehicle with Wing-Rudder Deflection
}

\author{
Y.H. Fan \\ School of Astronautics \\ North-western Polytechnic University \\ Xi'an, China \\ P.P. Yan \\ School of Astronautics \\ North-western Polytechnic University \\ Xi'an, China
}

\author{
Y.F. Yu \\ School of Astronautics \\ North-western Polytechnic University \\ Xi'an, China \\ X. Li \\ Li Shan Micro-electronics Technology Institute \\ Xi'an, China
}

\begin{abstract}
The scramjet performance of air-breathing hypersonic vehicle is highly correlated with flight height, Mach and angle of attack (AOA). The violent disturbance of the AOA can cause the engine power off. Consequently, the maneuverability of hypersonic vehicle is strictly limited in the cruising period. A composite control system for an air-breathing hypersonic vehicle is presented. The hypersonic vehicle has a configuration with tailcontrol rudders and a set of deflectable wings installed nearby the center of gravity. During the cruising period, the precision of AOA is achieved by deflecting tail rudders, and the command of maneuverable acceleration is tracked by deflecting wings. In the period of endgame, the scramjet should be power off, and the limit of AOA does not exist, so the tail rudders and the wings can both deflect to achieve high maneuverable acceleration. A linear quadratic (LQ) track control algorithm with integrator is introduced to design the composite control system. Simulation results demonstrate that the composite control system has good performance in tracking $\mathrm{AOA}$ and acceleration command by respective deflection of tail rudders and deflectable wings in cruise and also can track high acceleration command by coincident deflection during endgame.
\end{abstract}

Keywords-composite; hypersonic; vehicle; wing; rudder

\section{INTRODUCTION}

Air-breathing hypersonic vehicle with scramjet propulsion can flight at speed $M a>5$ in near space, and this vehicle has potential for use in civil and military. However air-breathing hypersonic vehicle has the uniqueness of the tightly integrated engine-airframe configuration, which results in significant coupling between the propulsion and aerodynamics. The violent disturbance of angle of attack can affect the performance of scramjet propulsion; even can induce the engine to power off. Therefore, the control system design becomes very challenging and the maneuverability of the vehicle is severely limited while the engine is working on. A traditional tail rudder deflection does not actively control the engine inlet conditions which allow the engines to power on when maneuvering, especially during push-over maneuver which will result in negative angles of attack. Many researchers have focused on the precise attitude control of hypersonic vehicle [1] [2] [3] [7], but very few literatures have studied the maneuverability while air-breathing hypersonic aircraft cruising.

The goal of this paper is to present a new scheme to solve the problem of the maneuverability of the vehicle. The hypersonic vehicle in this work has a configuration with tailcontrol rudders [4] [5] [8] and a set of deflectable wings installed nearby the center of gravity [6] [9]. During the cruising period, the precision of AOA is achieved by the deflecting of tail rudders, and the maneuverable acceleration command is tracked by deflecting wings. So the precision of AOA and high maneuverability can both be achieved by the composite control system. In the period of endgame, the scramjet should be power off, and the limit of AOA does not exist, the tail rudders and the wings are both used to achieve high maneuverable acceleration.

\section{MODEL OF VEHICLE}

The configuration of air-breathing hypersonic vehicle is shown as Fig 1.

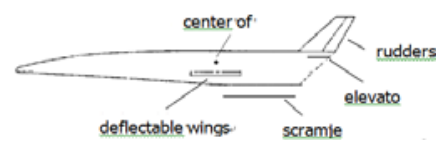

FIGURE I. CONFIGURATION OF VEHICLE.

The deflectable wings are installed near the center of gravity to produce additional lift without interaction moment while maneuvering, and the deflectable wings and elevators are not at the same level in order to avoid the couple induction. The position and efficiency of wings are carefully optimized by CFD computation.

The longitudinal model considered in this paper yields the nonlinear equations of motion of the vehicle as follow:

$$
\begin{aligned}
\dot{V} & =\frac{T \cos \alpha-D}{m}-g \sin (\theta-\alpha) \\
\dot{\alpha} & =\frac{-L-T \sin \alpha}{m V}+q+\frac{g}{V} \cos (\theta-\alpha) \\
\dot{q} & =\frac{M}{I_{y}} \\
\dot{\theta} & =q
\end{aligned}
$$


Where ${ }^{L}=\bar{q} S C_{L}, D=\bar{q} S C_{D}, M=\bar{q} S L_{r e f} C_{m} \cdot \bar{q}_{\text {denotes }}$ the dynamic press.

Let $x=[V, \alpha, q, \theta]^{T}, u=\left[\delta_{e}, \delta_{c}, \delta_{t}\right]^{T}$, equation(1) can be linearized as follow:

$$
\dot{x}=A x+B u, \quad y=C x, \quad z=H x+D u
$$

Where

$$
A=\left[\begin{array}{cccc}
X_{v} & X_{\alpha} & 0 & -g \\
Z_{v} & Z_{\alpha} & 1 & 0 \\
M_{v} & M_{\alpha} & M_{q} & 0 \\
0 & 0 & 1 & 0
\end{array}\right], \quad B=\left[\begin{array}{ccc}
X_{\delta e} & X_{\delta c} & X_{\delta t} \\
Z_{\delta e} & Z_{\delta c} & Z_{\delta t} \\
M_{\delta e} & M_{\delta c} & M_{\delta t} \\
0 & 0 & 0
\end{array}\right]
$$

Note that $\mathrm{z}$ is vector of output, $\mathrm{z}=\left[V, \alpha, N_{z}\right]^{T}$. Where $N_{z}=L_{\alpha} \alpha+L_{\delta c} \delta_{c}+L_{\delta e} \delta_{e}$, in cruise $\alpha=\alpha_{0}, \alpha_{0}$ denotes the trim angle of attack.

\section{COMPOSITE CONTROL SYSTEM DESIGN}

When the vehicle is in steady state, $\dot{x}(t)=0$, $x=x^{*}, u=u^{*}$,then the state equations is shown as follows[10]

$$
\left[\begin{array}{c}
x^{*} \\
u *
\end{array}\right]=\left[\begin{array}{cc}
A & B \\
M & N
\end{array}\right]^{-1}\left[\begin{array}{c}
0 \\
z *
\end{array}\right]=\left[\begin{array}{ll}
\bar{A}_{11} & \bar{A}_{12} \\
\bar{A}_{13} & \bar{A}_{14}
\end{array}\right]\left[\begin{array}{c}
0 \\
z *
\end{array}\right]
$$

Then

$$
x^{*}=\bar{A}_{12} z^{*}, \quad u^{*}=\bar{A}_{22} z^{*}
$$

Let $, \tilde{x}=x-x^{*}, \tilde{u}=u-u^{*}$, and define the track error, ${ }^{e=z_{c}-z}$,assuming $Z_{c}$ is a constant or slowly signal, an Integrate action is needed by augmenting the sate vector in order to get the zero steady state error.

$$
e_{I}=\int_{t_{0}}^{t} e(\tau) d \tau
$$

The augmented state equation is shown as follow:

$$
\left[\begin{array}{c}
\dot{\tilde{x}} \\
\dot{e}_{I}
\end{array}\right]=\left[\begin{array}{cc}
A & 0 \\
M & 0
\end{array}\right]\left[\begin{array}{c}
\tilde{x} \\
e_{I}
\end{array}\right]+\left[\begin{array}{c}
B \\
N
\end{array}\right] \tilde{u}
$$

Then the state equations can be written:

$\dot{x}_{\text {aug }}=A_{\text {aug }} x_{\text {aug }}+B_{\text {aug }} \tilde{u}, \quad y_{\text {aug }}=C_{\text {aug }} x$

The model following method is used to design the control system, considering the ideal model

$\dot{x}_{d}=A_{d} x, \quad y_{d}=C_{d} x$

Where $C_{\text {aug }}=I_{\text {aug }}, C_{d}=I_{d}$, then, define the error

$$
e_{a u g}=y_{a u g}-\mathcal{H}_{d}=\left(A_{a u g}-A_{d}\right) x_{a u g}+B_{a u g} Q \%
$$

Choose the LQ cost function
$J=\frac{1}{2} \int_{0}^{t}\left(e_{a u g}^{T} Q e_{a u g}+\psi_{0}^{T} R Q\right) d t$

Substituting (9) into (10), yields

$J=\frac{1}{2} \int_{0}^{t}\left(x_{\text {aug }}^{T} \hat{Q} X_{\text {aug }}+2 x^{T} \hat{S} \hat{\theta}_{0+} \theta^{T} \hat{R} \hat{R} \theta \partial\right) d t=\frac{1}{2} \int_{0}^{t}\left(\begin{array}{c}x_{\text {aug }} \\ \theta_{0}\end{array}\right)^{T}\left[\begin{array}{cc}\hat{Q} & \hat{S} \\ \hat{S}^{T} & \hat{R}\end{array}\right]\left(\begin{array}{c}x \\ \theta_{0}\end{array}\right) d t$

Where $\hat{Q}=\left(A_{\text {aug }}-A_{d}\right)^{T} Q\left(A_{\text {aug }}-A_{d}\right), \hat{S}=\left(A_{\text {aug }}-A_{d}\right) Q B_{\text {aug }}$, $\hat{R}=R+B_{\text {auq }}{ }^{T} Q B_{\text {aug }}$

Equation(11) is called LQ regulation, the control law for the augmented system can be computed as[10]

$$
\mathscr{U}^{\gamma}=-\hat{R}^{-1}\left[B^{T} P+\hat{S}^{T}\right] x_{\text {aug }}=-K x_{\text {aug }}
$$

Where $\mathrm{P}$ is the positive-definite solution to (for) the Algebraic Riccati equation

$$
A_{\text {aug }}^{T} P+P A_{\text {aug }}-\left(P B_{\text {aug }}+\hat{S}\right) \hat{R}^{-1}\left(B_{\text {aug }}^{T} P+\hat{S}^{T}\right)+\hat{Q}=0
$$

In consideration of the relationship between $u$ and $u^{*}$, we can get

$u=u^{*}-\ell(c$

Substituting (12) into (14), yields

$$
\begin{aligned}
& u=u^{*}-K x=u^{*}-K_{1}\left(x-x^{*}\right)-K_{2} e_{I} \\
& =\left(\bar{A}_{22}+K_{1} \bar{A}_{12}\right) z_{c}-K_{1} x-K_{2} e_{I}=K_{F} z_{c}-K_{1} x-K_{2} e_{I}
\end{aligned}
$$

The control law is implemented as in Fig 2.

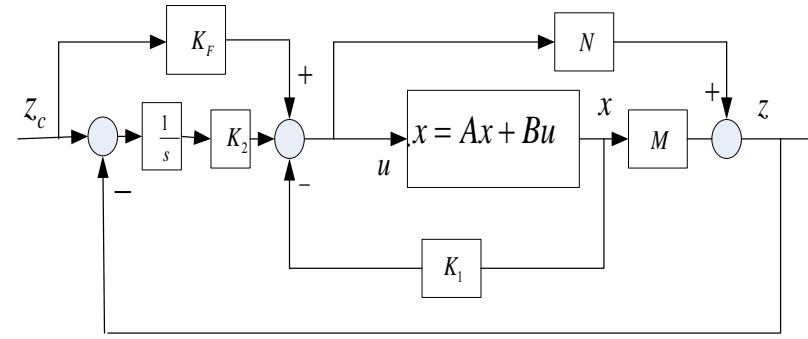

FIGURE II. THE CONTROL SYSTEM BLOCK DIAGRAM OF HYPERSONIC VEHICLE.

\section{SIMULATIONS OF COMPOSITE CONTROL SYSTEM}

\section{A. Simulations of Cruise}

Assuming the hypersonic vehicle flight height $H=25000 \mathrm{~m}$, the velocity $V=1460 \mathrm{~m} / \mathrm{s}\left(M_{a}=5.5\right)$, the trim angle of attack $\alpha=2 \mathrm{deg}$, and the command of the velocity $\left.V_{c}=1800 \mathrm{~m} / \mathrm{s}, M_{a}=6\right)$, and while the scramjet engine is power on, the command of the normal maneuver acceleration $N_{z c}=2 g$. The simulations of the composite control system are shown as follows. 


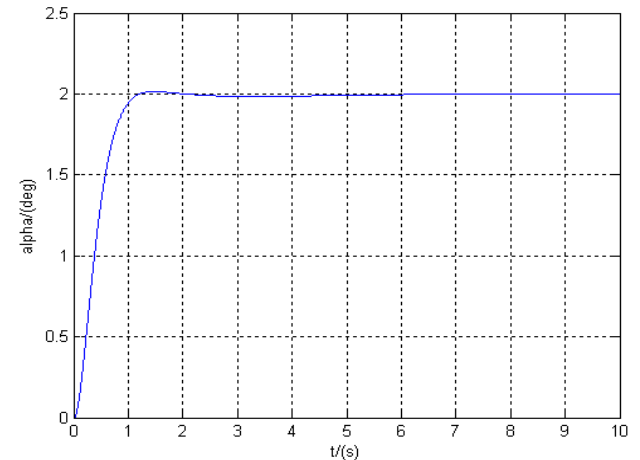

FIGURE III. RESPONSE OF AOA.

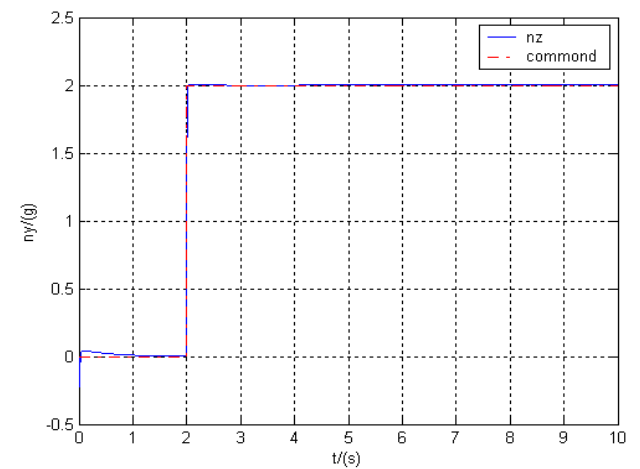

FIGURE IV. RESPONSE OF NORMAL ACCELERATION.

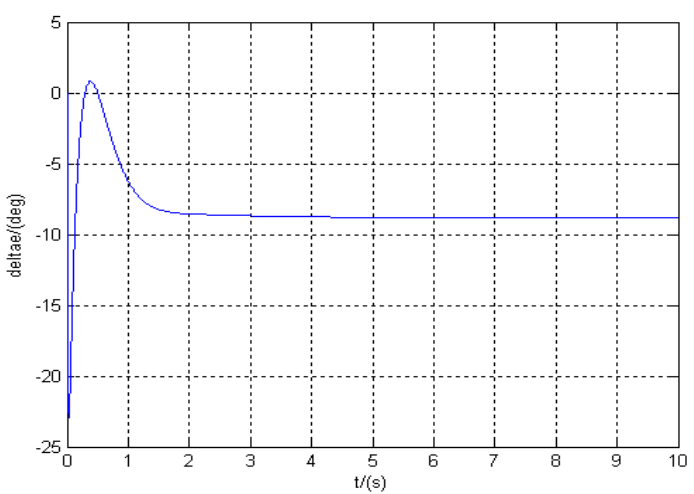

FIGURE V. RESPONSE OF ELEVATOR.

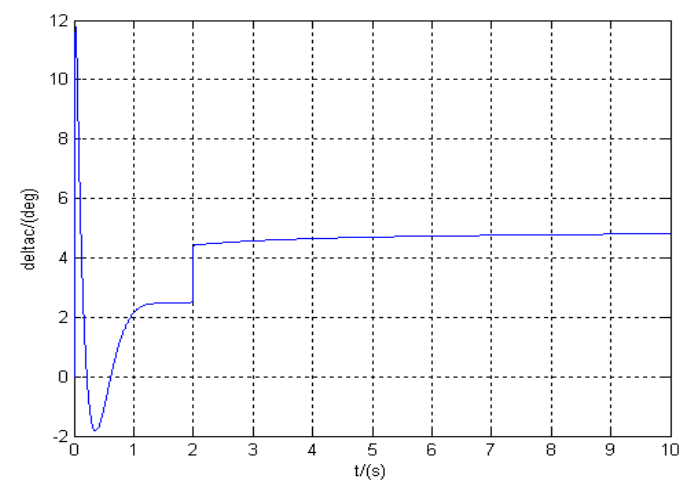

FIGURE VI. RESPONSE OF WING DEFLECTION.

\section{B. Simulations of Endgame}

Assuming the hypersonic vehicle flight height $H=25000 \mathrm{~m}$ the velocity $V=1860 \mathrm{~m} / \mathrm{s}\left(M_{a}=6\right)$. During the phase of endgame, the scramjet engine is power off, the tail rudders and the wings are both deflecting to trail high maneuverable acceleration command $N_{z c}=5 \mathrm{~g}$. The simulations of composite control system of hypersonic vehicle are shown as Fig.7 Fig.8. In these figures the result of only tail rudder deflection is contrast as dashed.

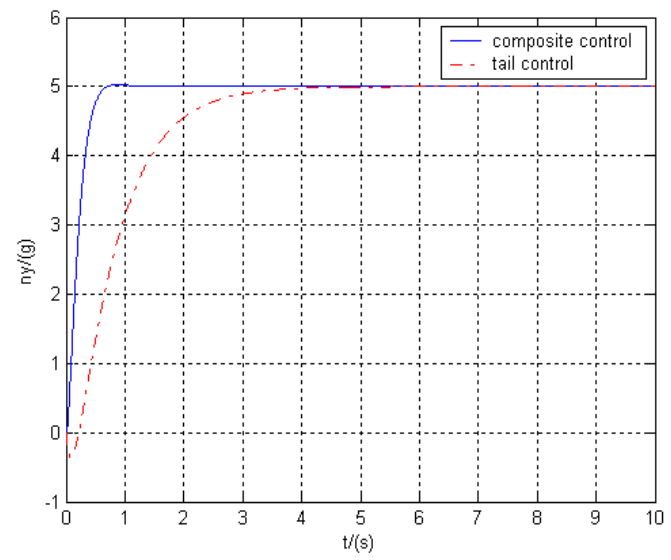

FIGURE VII. RESPONSE OF NORMAL ACCELERATION.

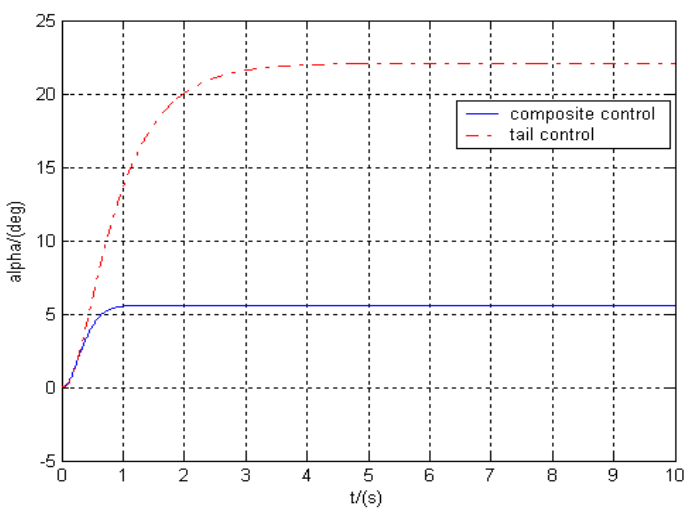

FIGURE VIII. RESPONSE OF AOA.

During the cruise, the composite control system can separately track the command of AOA and normal maneuver acceleration accurately as Fig3 Fig4. The AOA can be kept at trim state well while the hypersonic vehicle tracks normal maneuver acceleration command. Fig 4 and Fig 6 show that there is strong couple between AOA control channel and normal maneuver acceleration control channel.

From the results of simulation of endgame, the composite control system can track the command of $N_{z}$ more quickly than only tail rudder deflection as Fig 8.

So simulation results show that the composite control system with wings and rudder deflection not only can realize the maneuver flight while the scramjet working on, but also 
can meet the need of the high g normal maneuver acceleration during the endgame.

\section{CONCLUSION}

The new scheme to solve the problem of the maneuverability of the vehicle is feasible. The hypersonic vehicle has a configuration with tail control rudders and a set of deflectable wings installed nearby the center of gravity. During the phase of cruise, the AOA is not able to change tempestuously due to scramjet engine working conditions, consequently the AOA is achieved by tail rudders, and maneuverable acceleration command is tracked by deflecting wings. So the precision of AOA and maneuverability can both be achieved using composite control system while the engine working on. In phase of endgame, the scramjet should be power off, and the limit of AOA does not exist, the tail rudders and the wings can both deflect to achieve high maneuverable acceleration. Simulation results show that the composite control system can separately track the command of velocity, AOA and normal maneuver acceleration accurately, and the normal maneuver acceleration does not affect the AOA in cruise. During endgame the composite control system can track high maneuver acceleration command very quickly.

\section{ACKNOWLEDGEMENTS}

This paper is supported by Basic Research Foundation of Northwestern Polytechnic University under Grant No. JCY20130104 and this work are also supported by the Special Science Research Foundation of Doctor Subject for Higher Education under Grant No. 20136102120012.

\section{REFERENCES}

[1] Matthew Kuipers. Adaptive Control of an Aeroelastic Air-Breathing Hypers-onic Cruise Vehicle AIAA 2007-6326.

[2] Christopher I. Marrison, Robert F.Stengel. Design of Robust Control Systems for a Hypersonic Aircraft. JOURNAL OF GUIDANCE, CONTROL AND DYNAMICS, Vol.21, No.1, 1998

[3] H.Buschek and A.Calise. Hypersonic Flight Control System Design Using Fixed Order Robust Controllers. the proceeding of AIAA Sixth International Aerospace Plane and Hypersonic Technologies Conference.

[4] Michael A.Bolender, David B.Doman. Flight Path Angle Dynamics of Air-Breathing Hypersonic Vehicles. The proceeding of the 2006 AIAA Guidance, Navigation, and Control Conference, 24 Aug 06, Keystone.

[5] Torstens Skujins, Carlos E. S. Cesnik. Canard-Elevon Interactions on a Hypersonic Vehicle. JOURNAL OF SPACECRAFT AND ROCKETS Vol. 47, No. 1, 2010

[6] Phuong Vu. Daniel J.Biezad. Direct-Lift Design Strategy for Longitudinal Control of Hypersonic Aircraft. Journal of Guidance Control and Dynamics, 1994 Vol.17, NO.6.

[7] Lisa Fiorentini, Andrea Serrani. Nonlinear Robust Adaptive Controller Design for an Air-Breathing Hypersonic Vehicle Model. the Proceedings of the 2007 AIAA Guidance, Navigation and Control Conference.

[8] Michael W. Oppenheimer. Torstens Skujins. A Flexible Hypersonic Vehicle Model Developed with piston Theory. AIAA Atmospheric Flight Mechanics Conference and Exhibit August 2007, Hilton Head, South Carolina.

[9] R. F. Stengel, Flight Dynamics. Princeton, NJ: Princeton University Press, 2004.

[10] Stengel,R.F., Opitmal Control and Estimation, Dover Publications, New York, 1994. 